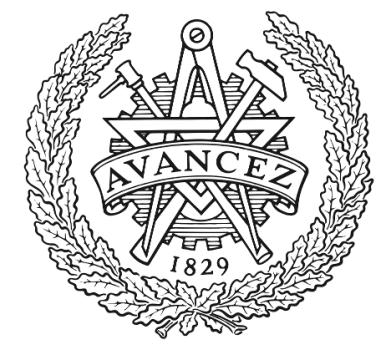

CHALMERS

UNIVERSITY OF TECHNOLOGY

\title{
Investigation of Phase-Lagged Boundary Conditions for Turbulence Resolving Turbomachinery Simulations
}

Downloaded from: https://research.chalmers.se, 2023-04-26 05:16 UTC

Citation for the original published paper (version of record):

Montero Villar, G., Lindblad, D., Andersson, N. (2020). Investigation of Phase-Lagged Boundary Conditions for Turbulence Resolving Turbomachinery

Simulations. AIAA AVIATION 2020 FORUM, 1. http://dx.doi.org/10.2514/6.2020-2982

N.B. When citing this work, cite the original published paper. 


\title{
Investigation of Phase-Lagged Boundary Conditions for Turbulence Resolving Turbomachinery Simulations
}

\author{
Gonzalo Montero Villar*, Daniel Lindblad ${ }^{\dagger}$ and Niklas Andersson ${ }^{\dagger}$ \\ Chalmers University of Technology, Gothenburg, SE-412 96
}

The present work explores whether phase-lagged boundary conditions can be used to perform scale resolving simulations of turbomachines. The phase-lagged approach considered is based on storing the flow signal, both at the pitch-wise boundaries and the rotor-stator interface, as its temporal Fourier coefficients for a finite number of harmonics. The method is implemented in an in-house CFD solver, G3D::Flow, which can perform both URANS and hybrid RANS/LES simulations. In order to evaluate the performance of the phase-lagged boundary condition, a comparison is made with a sliding mesh simulation on a compressor cascade. Furthermore, the possibility of breaking an error feedback loop generated in the sampling process by including multiple blade passages is also investigated. It is found that this approach greatly improves convergence and accuracy of the sampling.

\section{Nomenclature}

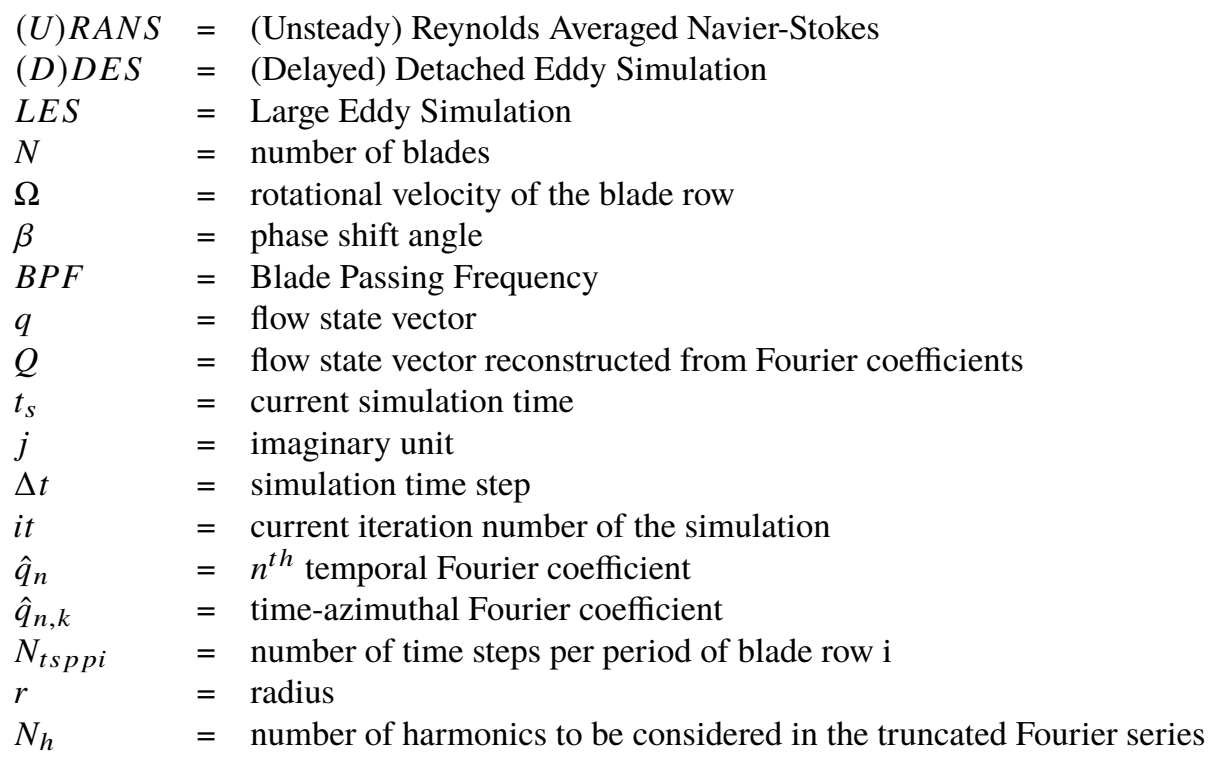

Subscripts

1,2 = blade-row index

*Ph.D. Student, Division of Fluid Dynamics, Department of Mechanics and Maritime Sciences, Chalmers University of Technology, SE-412 96 Gothenburg, Sweden

${ }_{\dagger}^{\dagger}$ Ph.D. Student, Division of Fluid Dynamics, Department of Mechanics and Maritime Sciences, Chalmers University of Technology, SE-412 96 Gothenburg, Sweden

Professor, Division of Fluid Dynamics, Department of Mechanics and Maritime Sciences, Chalmers University of Technology, SE-412 96 Gothenburg, Sweden 


\section{Introduction}

$\mathrm{C}^{\mathrm{OR}}$ the past decades, steady RANS computations with mixing-planes has been the most widely used approach to

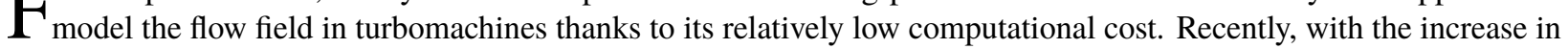
computational power, hybrid RANS/LES simulations, are however, becoming feasible. These methods are appealing since they have the potential of simulating complex unsteady flows with higher accuracy than URANS simulations, as well as help in the understanding of the underlying physics. In spite of technological advances, LES/DNS simulations are still computationally prohibitive nowadays for most industrial applications.

A commonly used hybrid RANS/LES method is the Detached Eddy Simulation (DES) introduced in [1]. The main idea behind DES is to simulate the attached boundary layer with an underlaying RANS model, and the rest of the domain with a LES model, using a unique formulation. This reduces the computational cost of the simulation by decreasing the grid resolution requirement for computing the flow inside the boundary layer. This method was further improved by the introduction of Delayed Detached Eddy Simulation (DDES) [2], which aimed at solving some of the issues related to grid sensitivity found on the original DES method.

In the particular case of turbomachinery simulations, it is often the case that two blade rows which are in relative motion to each other do not have matching pitches, i.e. the blade count is different. When steady state simulations are to be performed, this is not an issue, and one blade per row with a mixing-plane interface (where the tangentially averaged flow state at each radial span is transferred between blade rows) and periodic boundary conditions can be used. On the other hand, if unsteady simulations are computed, where for instance, the interaction between the wake of the first row and the second row is of interest (for example, tonal or broadband noise prediction), using a mixing-plane coupled with periodic boundaries is not adequate anymore, even for a case with equal pitches. To illustrate why this set up is not valid, Fig. 1 a) shows axial velocity contour of the wake generated by a rotating blade before it has gone through he mixing-plane interface, and Fig. 1 b) show what is being transferred to the downstream row through the interface. As can clearly be seen, by performing the circumferential averaging the wake structures are completely lost.

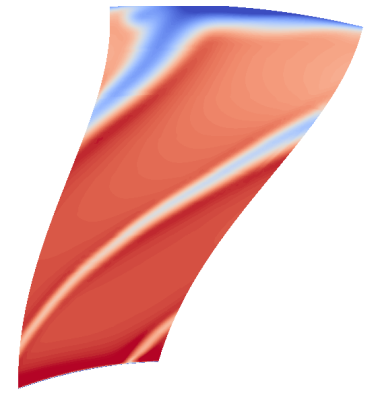

a)

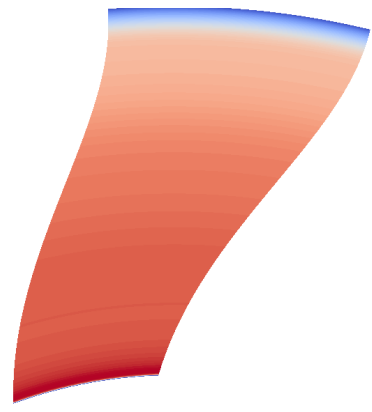

b)

Fig. 1 Axial velocity contours of a generic wake; a) wake before the mixing-plane averaging, b) flow transferred to the downstream blade by the mixing-plane interface.

An obvious solution to overcome the issue of the different pitches, is to simulate the entire $360^{\circ}$ wheel in both domains using a sliding grid interface. This would be the ideal scenario since all the flow features are transferred between both domains without any periodicity assumption. This makes it applicable to both URANS and turbulence resolving simulations, but unfortunately it is computationally prohibitive in most cases. If the simulation of the entire turbomachine needs to be avoided, two possible scenarios exist. In the first one, the pitch covered by each blade row domain is the same, which can be obtained in two different ways; if the blade count is the same only one blade is needed in each row, and if it is not, a combination of an integer number of blades in each row (usually low) that adds to the same pitch angle can be used (for instance 1 rotor blade and two stators in a 20 and 40 blade count configuration). The second scenario is where creating a combination that adds to the same pitch is not possible (or requires of too many blades). This prevents the possibility of using a combination of sliding grid with periodic boundary conditions.

If the flow only contains deterministic frequencies, an efficient alternative to time accurate, full annulus, simulations is the harmonic balance method [3, 4]. This method is based on representing the solution as a truncated Fourier series in time, with spatially varying coefficients. It then employs a discrete Fourier transform in order to either approximate the time derivative [3], or computes the Fourier coefficients of the nonlinear residual function [4]. This requires that the solution is stored either at a discrete set of time instances, or in terms of its Fourier coefficients. Although this 
increases the memory requirements of the method, the Fourier representation also enables the use of phase lagged periodic boundary conditions that can be used to reduce the computational domain to one blade per row. This, together with the fact that the harmonic balance method seeks directly for the steady state solution, makes it very efficient. Some notable work on the harmonic balance method can be found in [3-12]. Unfortunately, the harmonic balance method only works for cases that contain a set of deterministic frequencies. Therefore, it is not suitable for scale resolving simulation of turbulent flows. For this purpose, other methods needs to be used.

Other type of solutions are based on storing the flow signal at both the periodic boundaries and the blade-row interface for a whole period in order to be able to apply a phase-lagged condition on the other side of the interface/periodic boundary. Direct storage of all the flow quantities as proposed by Erdos et al. [13], although theoretically possible, usually becomes prohibitive due to storage requirements in grids with large number of cells, thus needing of some kind of data compression. One data compression approach is to sample the flow signal into temporal Fourier coefficients by making use of the assumption that the solution is periodic in time. One of such methods is based on the work by He [14] and Gerolymos et al. [15], referred to as the chorochronic method. This method has been successfully applied to different turbomachinery flow predictions [15-19].

Both the harmonic balance and the chorochronic methods are based on Fourier analysis. For a comprehensive review of the work done on these types of methods when applied to turbomachinery simulations over the past years, the reader is referred to the work by $\mathrm{He}$ [20].

More recently, Mouret et al. proposed a method where instead of compressing the data by decomposing it into Fourier coefficients, the data compression is carried out by performing a singular value decomposition of the flow signal [21]. Then, the singular values containing less energy and their corresponding left and right singular vectors are discarded, and only the more energy containing ones are stored for reconstructing the flow quantities. Mouret et al. applied their proposed method to the unsteady RANS simulation of the flow around a single-stage compressor [21].

In the present work, the chorochronic method is implemented in an in-house CFD solver, G3D::Flow. The implementation is validated and its performance tested in a one stage compressor rotor-stator configuration by performing both URANS and DDES simulations.

\section{Methodology}

Here the chorochronic method used, both for the pitch-wise boundaries as well as for the rotor-stator interface is described. The interested reader is referred to [15] for the complete derivation. The notation adopted here mostly follows the one used on [22].

\section{A. Chorochronic pitch-wise boundaries}

Let $N_{i}$ and $\Omega_{i}$ be the number of blades and rotational speed of the $i^{t h}$ blade row respectively. Based on these quantities, we can define the relative blade passing frequency for the first blade row as

$$
f_{1}=\frac{N_{2}\left|\Omega_{1}-\Omega_{2}\right|}{2 \pi}
$$

In order to compute the relative blade passing frequency of the second blade row, $f_{2}$, indexes 1 and 2 are simply swapped. This is the frequency at which a blade in a certain row sees the opposite row's blades, and hence the frequency related to the periodicity of the flow properties on the former blade row (leaving aside turbulent flow instabilities which might have their own frequency). Due to the missmatch in pitches between the two blade rows, two adjacent blades see the same flow field with a phase shift, $\beta$, that can be calculated as a function of the blade counts and rotational speeds as [15],

$$
\beta_{1}=-\frac{2 \pi\left(N_{1}-N_{2}\right) \operatorname{sign}\left(\Omega_{1}-\Omega_{2}\right)}{N_{1}}
$$

Again, in order to calculate the phase shift for the second blade row indexes must be swapped. This phase shift can be translated into a time shift as

$$
t_{\text {shift }_{i}}= \pm \frac{\beta_{i}}{2 \pi f_{i}}
$$

where the sign in Eq. 3 is changed depending on the tangential direction in which the phase shift is applied.

Due to the periodicity in time of the flow, it can be efficiently stored as a truncated Fourier series in time, where only $N_{h}$ harmonics are considered. For every cell at the pitch-wise boundary, the $n^{\text {th }}$ temporal Fourier coefficient is 
computed as follows,

$$
\begin{aligned}
& \hat{q}_{n}=f_{1} \int_{t_{s}-\frac{1}{f_{1}}}^{t_{s}} q(t) e^{-2 j \pi n t f_{1}} d t \\
& \hat{q}_{n}=f_{2} \int_{t_{s}-\frac{1}{f_{2}}}^{t_{s}} q(t) e^{-2 j \pi n t f_{2}} d t
\end{aligned}
$$

where $t_{s}$ is the current time of the simulation, $j$ is the imaginary unit, $q(t)$ are the flow variables at time $t$ in a given cell, and the first or the second equation in Eq. 4 will be used depending which blade row the cell belongs to. Due to the discrete nature of CFD methods, Eq. 4 can be rewritten as,

$$
\begin{aligned}
& \hat{q}_{n}=f_{1} \sum_{i=i t-N_{t s p p 1}}^{i t} q(i \Delta t) e^{-2 j \pi n i \Delta t f_{1}} \Delta t \\
& \hat{q}_{n}=f_{2} \sum_{i=i t-N_{t s p p 2}}^{i t} q(i \Delta t) e^{-2 j \pi n i \Delta t f_{2}} \Delta t
\end{aligned}
$$

where $N_{\text {tsppi }}$ is the number of time steps per period associated to the frequencies computed for the $i^{t h}$ blade row with Eq. 1. $\Delta t$ is the simulation time step and $i t$ is the current iteration of the simulation.

In order to avoid storing the time signal over one full time period before the temporal Fourier coefficients in Eq. 5 can be computed, a moving average technique is used to update them [15]. This technique updates the Fourier coefficients by adding the contribution of the current flow solution to all coefficients and subtracting the value one period back in time obtained using the coefficients. By using this updating technique, one can clearly see that when it has fully converged, due to the periodicity of the signal, the reconstructed value one period back in time and the current value should be the same, hence the Fourier coefficients remain unchanged. This moving average based technique can be expressed as,

$$
\begin{aligned}
& \Delta \hat{q}_{n}=\frac{1}{N_{t s p p 1}}\left(q\left(t_{s}\right)-Q\left(t_{s}-\frac{1}{f_{1}}\right)\right) e^{-2 j \pi n t_{s} f_{1}} \\
& \Delta \hat{q}_{n}=\frac{1}{N_{t s p p 2}}\left(q\left(t_{s}\right)-Q\left(t_{s}-\frac{1}{f_{2}}\right)\right) e^{-2 j \pi n t_{s} f_{2}}
\end{aligned}
$$

where $\Delta \hat{q}_{n}$ is the update of the $n^{t h}$ temporal Fourier coefficient, and $Q(t)$ is the value obtained when reconstructing the flow properties at time $t$ using the temporal Fourier coefficients computed with Eq. 5 . Note that the subtraction in Eq. 6 is only done when the simulation time exceeds one time period associated with the relative blade passing frequency of the corresponding blade row computed with Eq. 1.

Finally, when values are needed in order to transfer information between both sides of the pitch-wise boundaries, the temporal Fourier coefficients are used to reconstructed the desired flow properties with the corresponding time shift computed according to Eq. 3 as,

$$
Q\left(t_{s}+t_{\text {shift }_{i}}\right)=\sum_{n=-N_{h}}^{N_{h}} \hat{q}_{n} e^{2 j \pi n f_{i}\left(t_{s}+t_{\text {shift }_{i}}\right)}
$$

\section{B. Chorochronic blade-row interface}

When dealing with a blade-row interface, the interacting spinning modes that occur can be described by the well known Tyler-Sofrin modes [23],

$$
m_{n, k}=n N_{1}+k N_{2}
$$

These modes spin at the following frequencies in the first and second blade row's frame of reference respectively,

$$
\begin{aligned}
& \omega_{n}=n N_{2}\left(\Omega_{1}-\Omega_{2}\right) \\
& \omega_{k}=k N_{1}\left(\Omega_{2}-\Omega_{1}\right)
\end{aligned}
$$

Due to the double periodicity in space and time, the flow signal is represented as a truncated double Fourier series, where only $N_{h}$ harmonics are considered. In order to obtain the time-azimuthal Fourier coefficients, first the temporal 
coefficients are computed using a moving average technique at every cell at the interface. This is done in the same manner as for the pitch-wise boundaries, as described in Sec. III.A. Then the time-azimuthal coefficients can be constructed for each radial span from the time Fourier coefficients as,

$$
\begin{aligned}
& \hat{q}_{n, k}(r)=\frac{N_{1}}{2 \pi} \int_{0}^{\frac{2 \pi}{N_{1}}} \hat{q}_{n}(\theta, r) e^{j m_{n, k} \theta} d \theta \\
& \hat{q}_{n, k}(r)=\frac{N_{2}}{2 \pi} \int_{0}^{\frac{2 \pi}{N_{2}}} \hat{q}_{k}(\theta, r) e^{j m_{n, k} \theta} d \theta
\end{aligned}
$$

for each of the blade row respectively. Finally when the reconstructed values are needed in order to transfer information between blade rows, the necessary flow properties for each of the blade rows respectively can be obtained using the time-azimuthal Fourier coefficients computed using Eq. 10 as,

$$
\begin{aligned}
& Q(r, \theta, t)=\sum_{n=-N_{h}} \sum_{k=-N_{h}}^{N_{h}} \hat{q}_{n, k}(r) e^{j \omega_{n} t-j m_{n, k} \theta} \\
& Q(r, \theta, t)=\sum_{n=-N_{h}}^{N_{h}} \sum_{k=-N_{h}}^{N_{h}} \hat{q}_{n, k}(r) e^{j \omega_{k} t-j m_{n, k} \theta}
\end{aligned}
$$

\section{Periodic boundary decoupling assessment}

In order to validate the implementation of the method in the in-house G3D::Flow solver, an initial test case is used, a straight cascade where a vortical gust is introduced at the inlet. For this simpler case three simulations are run, the first one with a pitch that matches the tangential wave number of the gust, hence allowing for standard periodic boundary conditions (this case will be used as the benchmark), and the other two using chorochronic periodic boundary conditions. The reason why two chorochronic simulations are run, is because different strategies for the periodic set up are used in each of them. In the first one, only one sector is simulated (Fig 2 a)), whereas on the second one three sectors will be used (Fig 2 b)). As discussed later, the second strategy aims at improving the stability and convergence of the Fourier coefficients by breaking an error feedback loop.

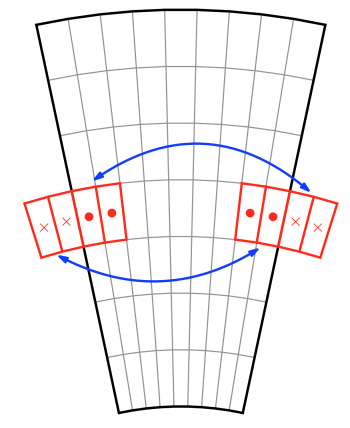

a)

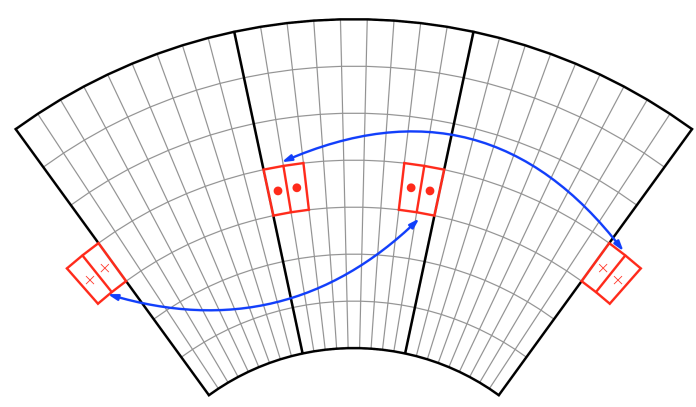

b)

Fig. 2 Periodic boundary set up strategy. a) single sector with coupled periodic boundaries, b) multiple sectors with decoupled periodic boundaries. ○ represents the cells where the temporal Fourier coefficients are sampled and $\mathrm{X}$ the ghost cells where the phase shifted values are introduced. The blue arrows show which Fourier coefficients are used for phase shifting which values.

The strategy shown in Fig. 2 b) is used for two reasons. Firstly, it helps with the stability and convergence of the temporal Fourier coefficients. This is because in the standard setting (see Fig. 2]a)), there is an underlying feedback loop between both sides of the periodic interface that delays the convergence of the moving average. By looking at the standard set up, it can be seen that when the Fourier coefficients are not converged, an error is introduced when they are used to introduce phase lagged values on the ghost cells of the left side. This introduced error is going to propagate via the flux calculation to the sampling nodes on the left side, which in turn, introduces an error on the nodes on the right 
side when the left side Fourier coefficients are used to calculate the phase-lagged values on right side. This ongoing loop deteriorates the convergence and the stability of the moving averaged used to update the Fourier coefficients. By using the decoupled periodic boundary strategy (Fig. 2 b)) this feedback loop is broken as an error in sampling on one side does not directly introduce an error on the other side. The second reason why the decoupled boundary strategy is used, is to allow for some non periodic features of the flow to occur in the middle sector, which is specially interesting when DDES simulations are performed, as done in [24].

The same decoupling (breaking of the error feedback loop) effect could be achieved by employing only two sectors (as will be done later on the rotor domain, see Fig. 6, and similar to [18, 25] where a double passage strategy was used int order to improve the quality of the sampled flow signal and convergence improvements were reported), but due to the later reason of wanting non-periodic features, three sectors have been chosen. When using this strategy the algorithm for the chorochronics periodic boundaries does not change. The frequency of the flow features is still computed according to Eq. 1. and the only change occurs in the phase shift and corresponding time shift defined in Eq. 2 and Eq. 3. which are now doubled to compensate for the extra sector that the solution needs to be phase shifted.

Figure 3 shows the axial velocity at the same time instance for the three aforementioned cases with the inlet gust; the standard periodic, the standard chorochonic periodic and the decoupled boundary periodic chorochronic. In the chorochronic simulations the phase shift applied on the periodic boundaries can be clearly appreciated. But in order to make a more quantitative comparison, sampling is performed along an horizontal line (at one third of the height of the domain shown in Fig. 3 a)). The results of this line plot can be seen in Fig. 4 where an excellent agreement can be seen for both type of periodic configurations.

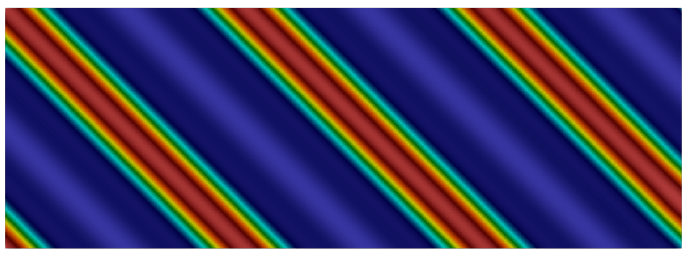

a)

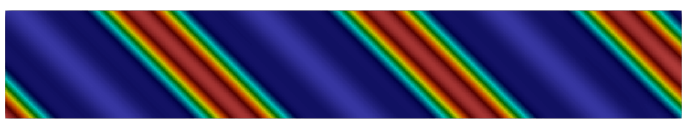

b)

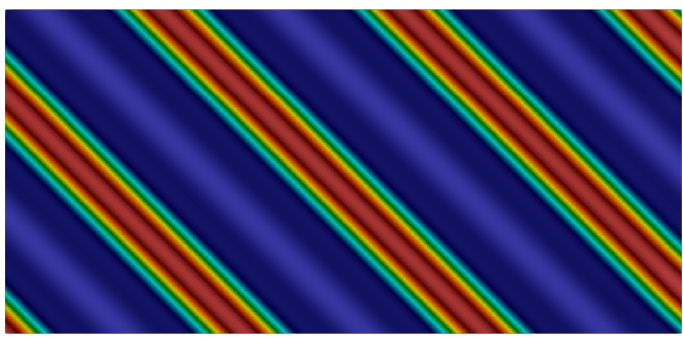

c)

Fig. 3 Chorochronic periodic validation and periodic decoupling assessment. Axial velocity contours; a) standard periodic, b) standard chorochronic periodicity and c) decoupled chorochronic periodicity. Same colorbar in all three contour plots. 


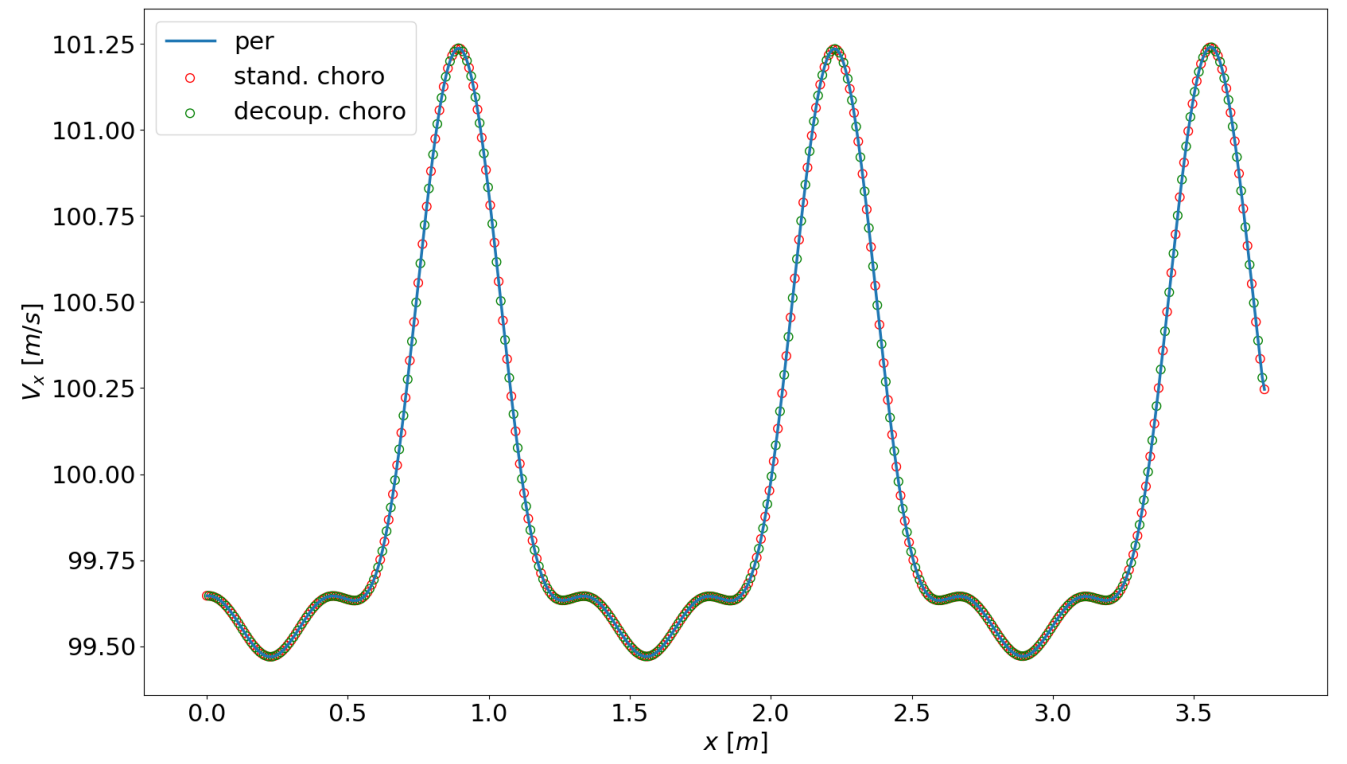

Fig. 4 Axial velocity comparison along a horizontal line. Note that for visibility enhancement not all the points have been plotted for standard and decoupled chorochronic, and they have been alternated.

Finally, in order to confirm the hypothesis that decoupling the periodic boundaries when using chorochronic boundary conditions helps the convergence and stability of the Fourier coefficients update, the convergence of the moving average along both chorochronic simulations just presented is measured and compared. The convergence of the error of the moving average is computed as the error when comparing the current flow state with the one predicted one period back in time using the temporal Fourier coefficients;

$$
\epsilon=\frac{q\left(t_{s}\right)-Q\left(t_{s}-\frac{1}{f}\right)}{q\left(t_{s}\right)}
$$

where $q\left(t_{s}\right)$ is the flow variable at the simulation physical time, and $Q\left(t_{s}-1 / f\right)$ is the value obtained by reconstructing the same flow variable one period back in time using the temporal Fourier coefficients. In order to be able to compare both the standard and decoupled chorochronic simulations, the value plotted in Fig. 5is the maximum $\epsilon$ over the entire interface at each time step. It can clearly be seen how decoupling the periodic boundaries helps and improves the convergence of the moving average. Note that the two line plots on Fig. 5 do not start at simulation time zero, the reason for this is that as previously mentioned in Sec. III.A when describing Eq. 6, the subtraction part of the moving average is not done until the simulation time has reached one time period.

\section{Computational set up}

In order to solve the Favre-averaged Navier-Stokes equations, the in-house solver G3D::Flow is used, which is based on the family of codes developed by Eriksson [26]. G3D::Flow is a finite volume CFD solver for compressible flows developed and maintained at the division of Fluid Dynamics at Chalmers University of Technology. For advancing the solution in time a three-stage second order accurate Runge-Kutta algorithm is employed. Convective fluxes are computed with a third order accurate upwind biased scheme, whereas the diffusive ones are evaluated with second order accurate centered scheme. Regarding the turbulence modeling, when performing URANS simulations, Spalart-Allmaras turbulence model is used [27], and for turbulence resolving simulations, DDES with Spalart-Allmaras as underlying RANS model is used with a shear-layer-adaptive length scale [28, 29].

In the literature, some authors reported a non ideal behaviour of the shielding function in the original DDES model (usually related to mesh resolution) [30-32]. The shielding function is the function that switches between RANS and 


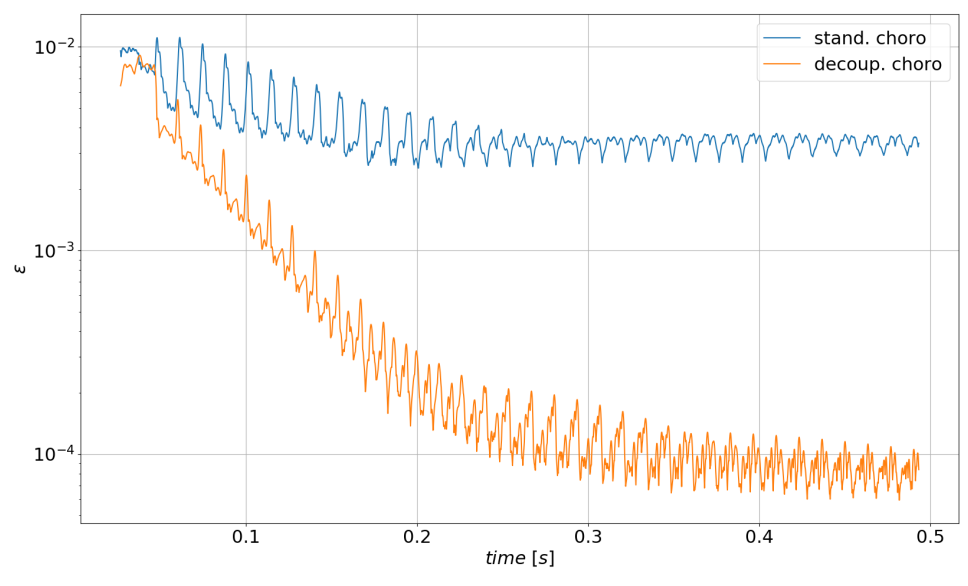

Fig. 5 Moving average convergence comparison between both chorochronic periodic strategies.

LES modes in the DDES model. In this work the modified shielding function proposed in [32] and implemented in [31] is used. Since the main purpose of this work is not to asses the performance of the DDES model or how well its shielding function can transition between the RANS and LES regions, identical meshes (one rotor and one stator mesh are generated, copied and rotated as many times as necessary) and numerics (except the periodic and rotor-stator interface boundary conditions) will be used to eliminate ambiguity when evaluating the performance of the phase-lagged boundary conditions.

In order to asses the performance of the phase-lagged boundary conditions described in Sec. [II] a sector of a slightly modified version of the VINK compressor R1S1B will be used [33, 34] (this sector covers approximately 15\% of the span of the blades and is located at mid span). The modifications include having 50 rotor and 90 stator blades instead of the 51 and 88 of the original design. This change has been made so that a 5 rotor and 9 stator simulation can be run with standard periodic boundary conditions and sliding mesh as blade-row interface. This set up is the one used as a benchmark and the one to which the phase-lagged simulations are compared to.

In the phase-lagged simulations, the decoupling of the periodic boundaries explained in Sec. III.C is sought for, therefore, 2 rotor blades and 3 stator blades will be accounted for. Figure 6 shows a schematic of the set up used for the phase-lagged simulations. The thick black lines represent the boundaries of each blade's domain, the arrows show how the connection between the chorochronic boundaries is set up and what phase shift angle is applied in each of them. The areas shaded with red vertical lines in Fig. 6represent the cells that will be used to sample the temporal Fourier coefficients, and the areas shaded by blue diagonal lines, the ghost cells where the phase shifted values are introduced. The points marked as $P 1, P 2$ and $P 3$ represent the points where flow data is sampled.

\section{Results}

\section{A. Matching pitches with sliding mesh and standard periodicity}

As a benchmark to compare the phase-lagged simulations against, the case where 5 rotor blades and 9 stator blades are simulated, allowing for the use of a sliding mesh and standard periodic boundary conditions, will be used. Thus, the first thing to do is to verify that the sliding mesh implemented in G3D::Flow is working as intended. In order to do so, variables are sampled along a constant radius line right before the interface, in the rotor domain, and right after the interface, on the stator domain. Figure 7 shows the result obtained for the axial velocity component on the URANS simulation, where a good agreement can be seen. The same quality of agreement is observed for the DDES simulation and also for the rest of the variables conforming the flow state, but are not presented here for brevity. 


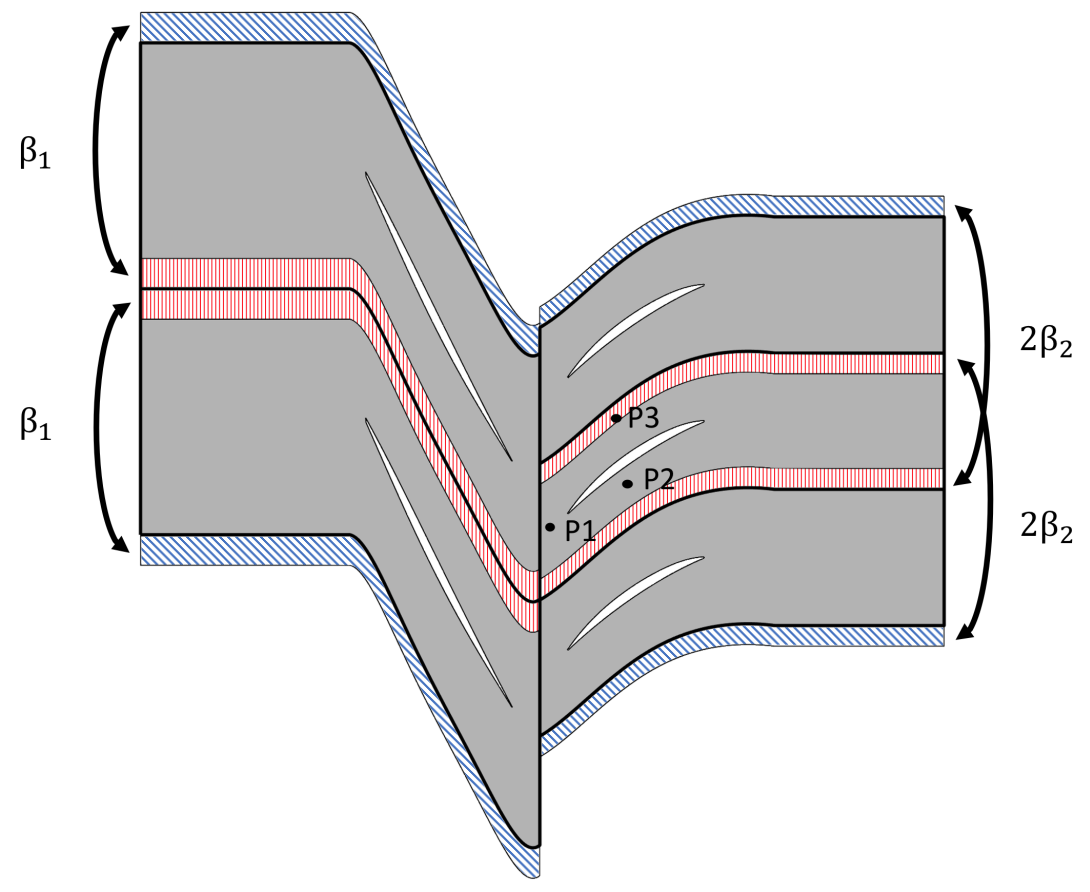

Fig. 6 Computational set up with corresponding phase shift angles and periodic boundary decoupling schematic. The points marked $P 1, P 2$ and $P 3$ are data sampling points.

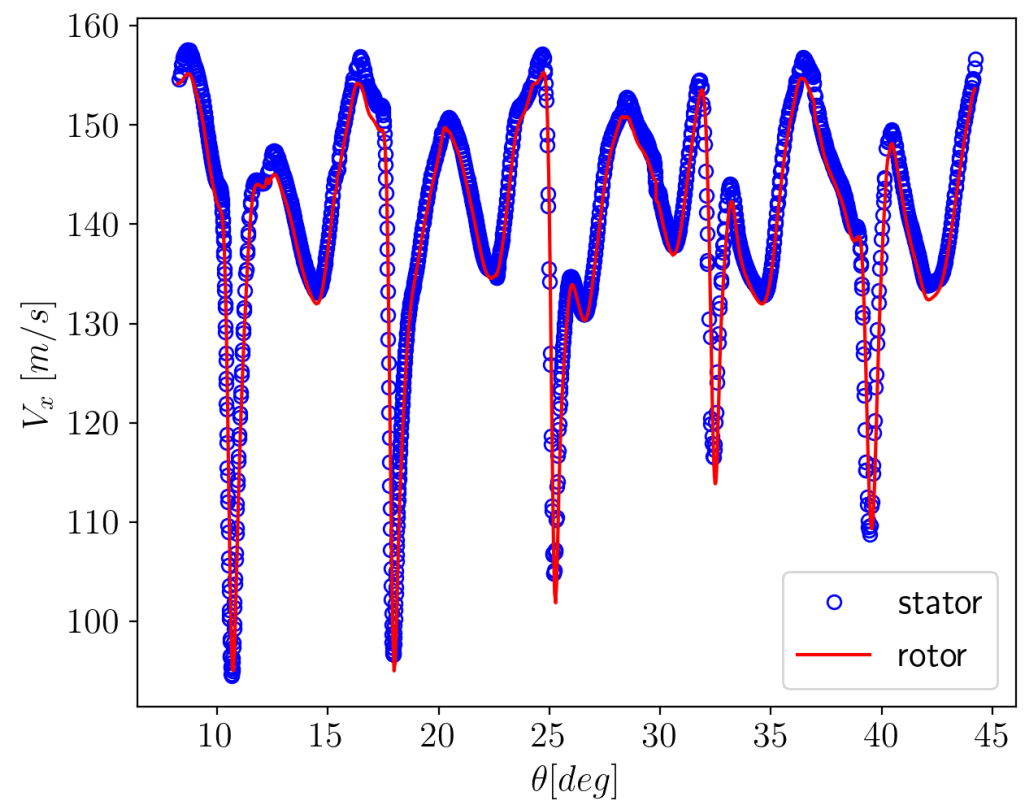

Fig. 7 Comparison of axial velocity before and after the sliding mesh interface.

\section{B. Chorochronic URANS}

Here the results obtained using chorochronic periodicity and rotor-stator interface are compared with the ones obtained with the sliding mesh. For this simulation the number of harmonics accounted for was set to 20, which was considered to be sufficient given the results presented later (Gerolymos, for instance, reported successful results for 
an axial compressor and a fan stage using 10 harmonics [16]). A total of 20 periods $\left(f_{2}^{-1}\right)$ were simulated, reaching converged temporal coefficients both at the rotor-stator interface and the pitch-wise boundaries at around time period number 13. Figure 8 shows instantaneous entropy contours for both the sliding mesh and chorochronic simulation at the same time instance, where a qualitatively good agreement can be seen.

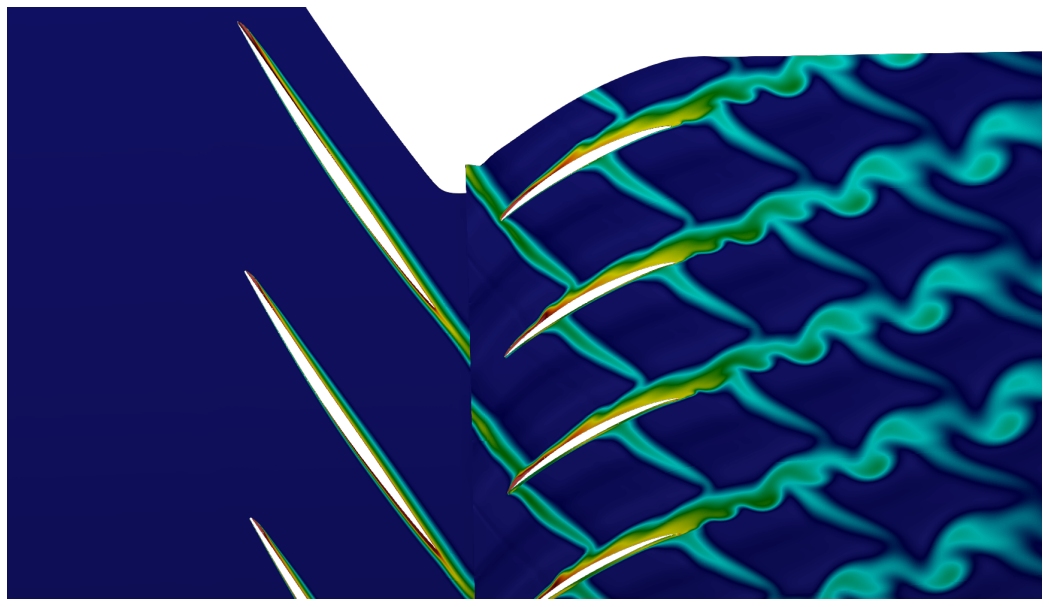

a)

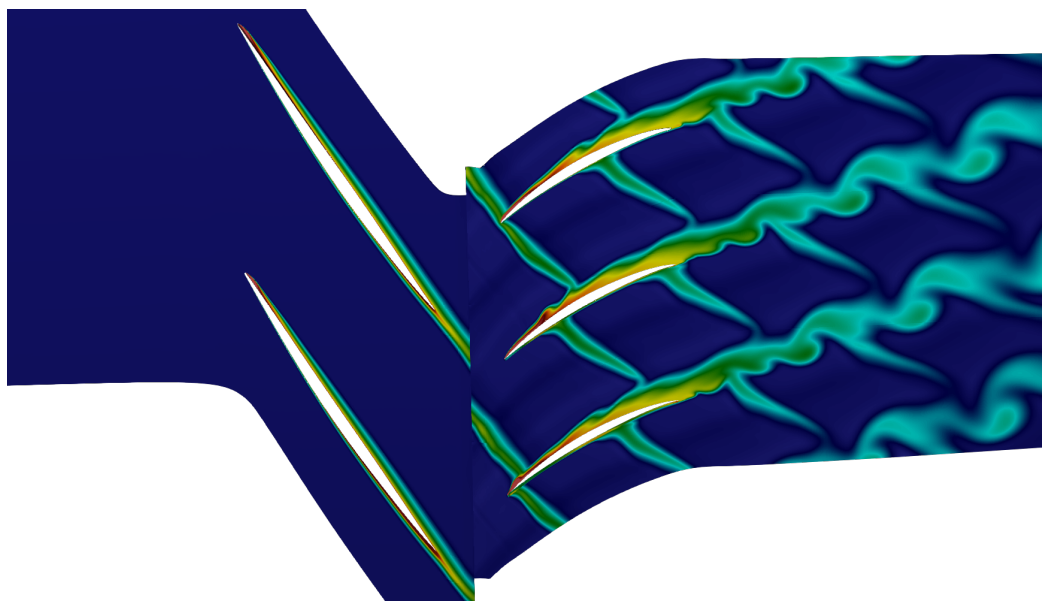

b)

Fig. 8 Entropy contours of URANS simulation a) sliding mesh and standard periodicity, and b) chorochronic periodicity and rotor-stator interface. Same colorbar.

In order to analyze the performance of the phase-lagged boundary conditions in more depth, data is sampled at three points (see Fig. 6). Figure 9 shows the comparison between the URANS simulations where sliding mesh with standard periodicity are used and the one where chorochronic interface and periodicity is employed. As can be seen from the figure, the agreement of the axial velocity profiles sampled at the three points is quite good, as well as the energy content of those. Table 1 confirms the good agreement by showing the mean, maximum and standard deviation of the percentage error with respect to the sliding mesh computation at the three sampling points. This is within the expectations, as most of the energy is contained on frequencies that are multiple integers of the BPF which is exactly what the chorochronic boundary conditions are sampling and transferring on both the rotor-stator and periodic interfaces (see right hand side plots on Fig. 9].

\section{Chorochronic DDES}

In this section a similar analysis as the one presented for the results obtained with URANS simulations is carried out for the performed DDES computations. In this case, the number of harmonics sampled and transferred by the 


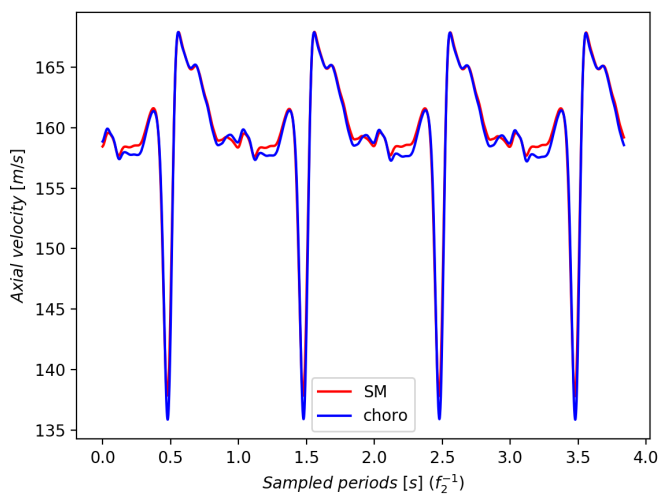

a)

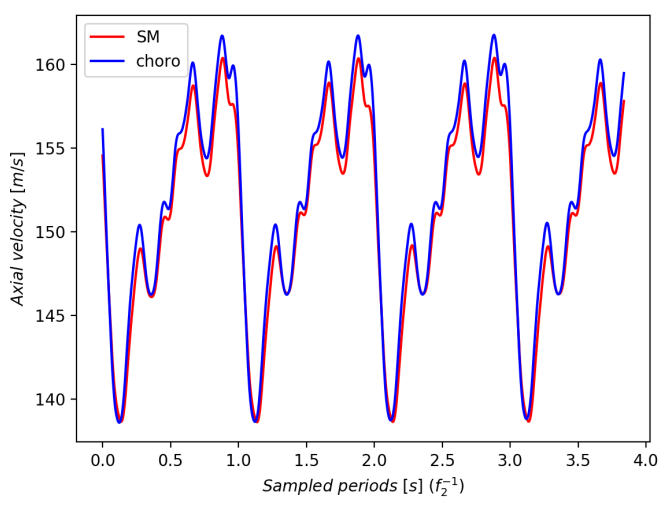

c)

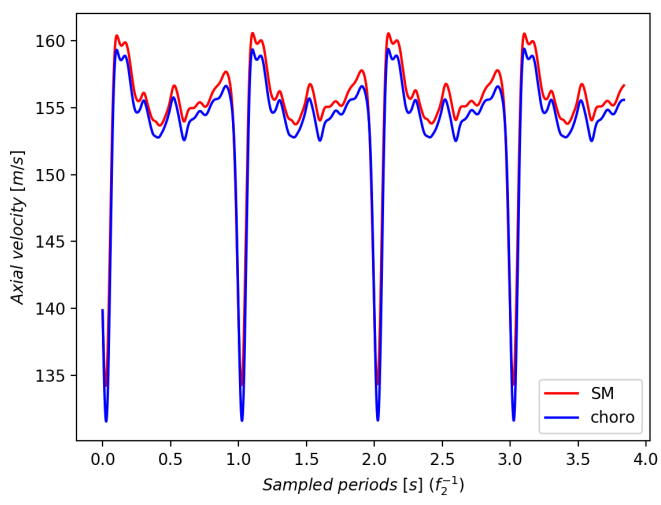

e)

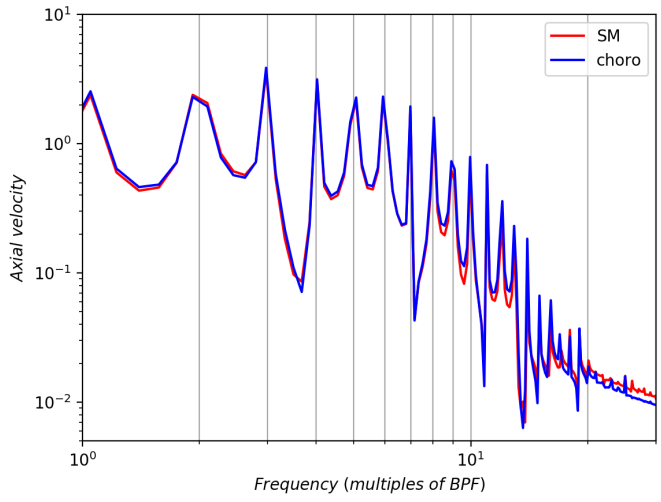

b)

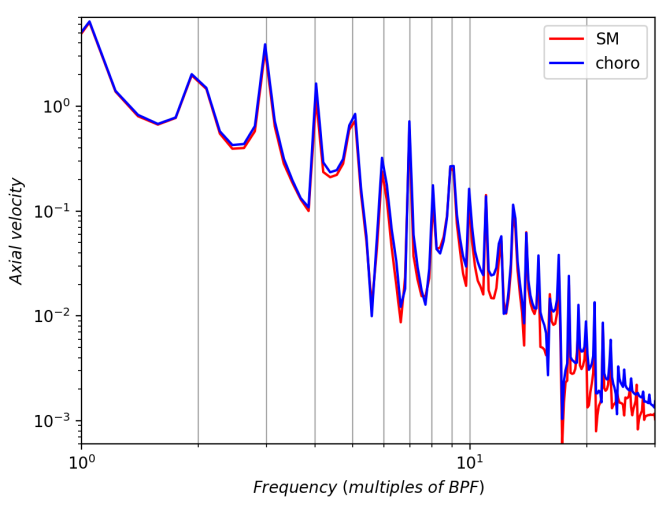

d)

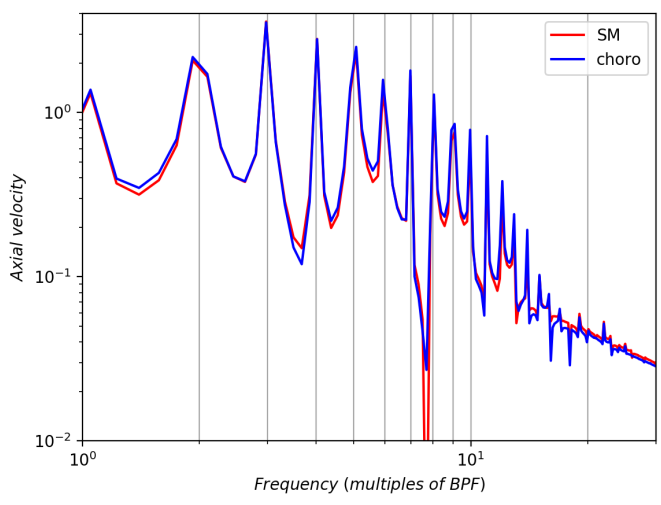

f)

Fig. 9 Comparison of sampled data gathered on points illustrated in Fig. 6 for the URANS simulations. SM and choro refer to the sliding mesh and chorochronic simulation respectively. Axial velocity and its frequency content shown for each point. a) and b) point 1, c) and d) point 2 and e) and f) point 3.

chorochronic boundary conditions is increased from 20 to 30. Similarly as for the URANS simulations, a total of 20 periods $\left(f_{2}^{-1}\right)$ were simulated, but in this case the convergence of the Fourier coefficients was delayed 2 extra time periods, needing a total of 15 . Figure 10 shows instantaneous entropy contours for the DDES simulations for both the 
Table 1 Percentage error for the URANS chorochronic simulation's sampled axial velocity.

\begin{tabular}{c||c|c|c} 
& mean & max & std \\
\hline \hline P1 & 0.28 & 1.83 & 0.31 \\
P2 & 0.67 & 1.60 & 0.37 \\
P3 & 0.72 & 2.64 & 0.39
\end{tabular}

sliding mesh and chorochronic simulations at the same time instance. Even though a qualitatively good agreement can be seen, there are some features that are worth noticing from these figures. By taking a look the rotor wake impacting the third stator blade, it can be seen, how on the sliding mesh simulation (Fig. 10 a)), the vortices shedding from the rotor blade are transferred from the rotor domain into the stator domain across the interface. These vortices have their own frequency which is not coupled to the BPF. Thus, by looking at the same location on the chorochronic simulation (Fig. 10 b)) one can clearly see how the Fourier sampling performed at the interface has filter the vortices shedding from the rotor blades out, and has not been able to transfer them into the stator domain, resulting in a much smoother wake.

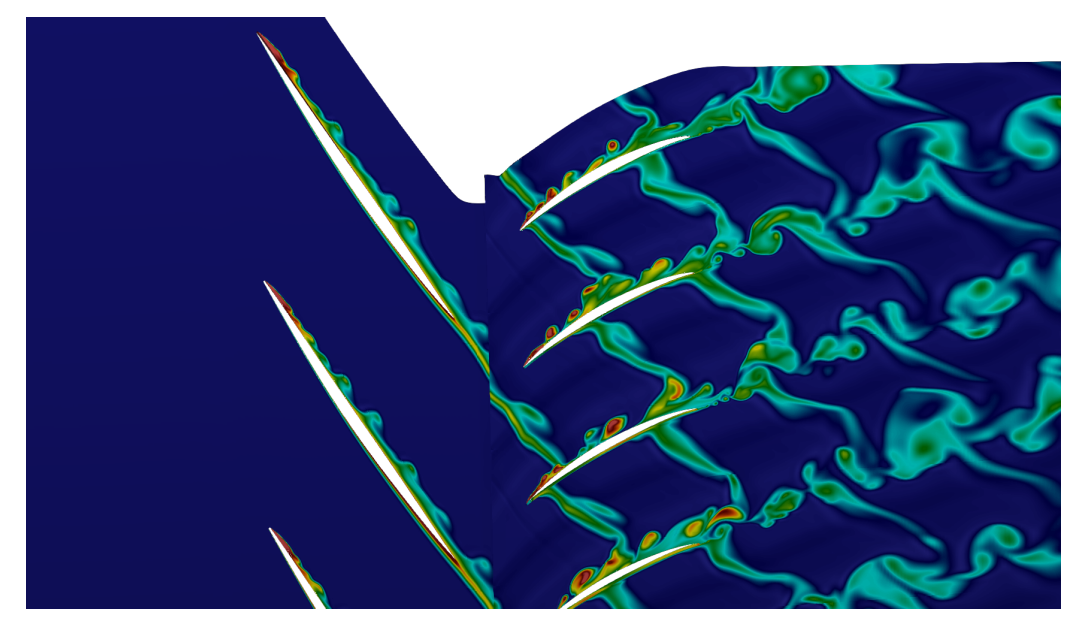

a)

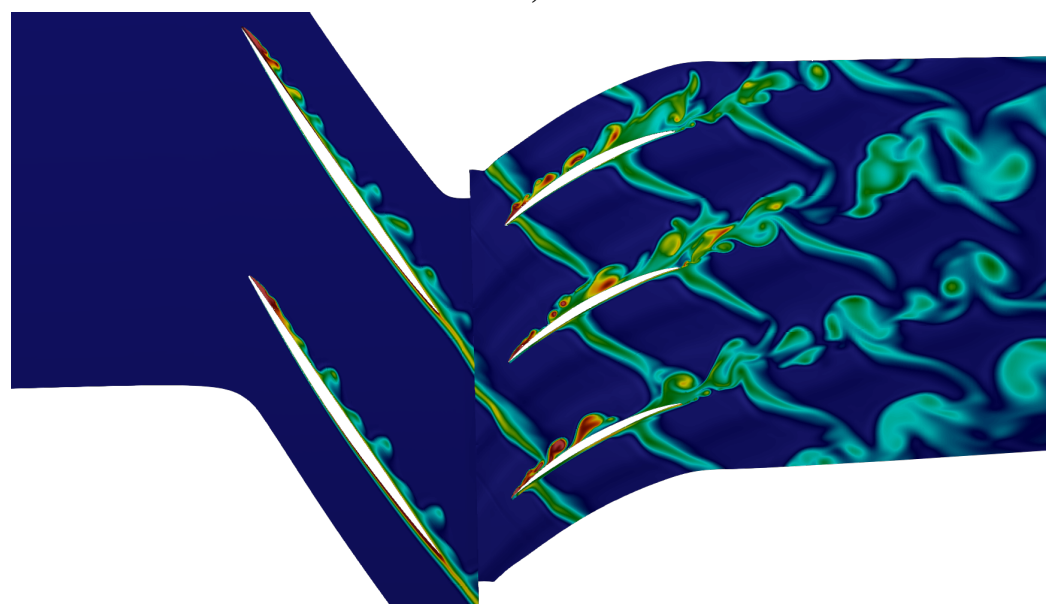

b)

Fig. 10 Entropy contours of DDES simulation a) sliding mesh and standard periodicity, and b) chorochronic periodicity and rotor-stator interface. Same colorbar.

Data is sampled at the same three points as for the URANS case (see Fig. 6). The comparison is performed using the DDES sliding mesh simulations as a benchmark case. The results for the time evolution of the axial velocity as well 
as its energy content at the sampling points can be seen in Fig. 11. As can be see from the figure, the agreement on the axial velocity profiles is not quite as good as it was for the case of the URANS simulation. Table 2 verifies this by showing the mean, maximum and standard deviation of the percentage error, where maximum errors close to $10 \%$ can be found. In order to understand why this mismatch occurs, one can take a look at the spectrums (see plots on the right hand side of Fig. 11p. Here quite a good agreement can be seen for the frequencies directly correlated to the BPF for the three different sampling points, meaning that the chorochronic boundary conditions were able to sample and transfer those across the different interfaces. Once higher frequencies are analyzed, it is quite clear the energy on those frequencies is not retained anymore and both curves start to deviate from one another. This can clearly be seen by taking a look at the end of the spectrums presented in Fig. 11

Table 2 Percentage error for the DDES chorochronic simulation's sampled axial velocity.

\begin{tabular}{c||c|c|c} 
& mean & $\max$ & std \\
\hline \hline P1 & 1.73 & 9.78 & 1.44 \\
P2 & 1.37 & 9.13 & 1.38 \\
P3 & 1.88 & 5.56 & 1.08
\end{tabular}

\section{Conclusions}

In the current work the phase-lagged periodic and interface boundary conditions based on the chorochronic approach are presented and implemented in the in-house compressible CFD code, G3D::Flow. A strategy for decoupling the periodic boundaries that improves the convergence and stability of the Fourier coefficients is described and tested.

The newly implemented boundary conditions are used to perform both URANS and DDES simulation on a span-wise sector of a one stage compressor, and their results compared with results obtained running sliding mesh and standard periodicity on a case where the pitches on both domains match. The URANS simulations show very good agreement, whereas the DDES simulations, despite of being able to capture the overal trend, are not able to retain the energy contained in frequencies uncorrelated to the blade passing frequency, thus deteriorating the solution.

The fact that only a span-wise sector is simulated might have given the phase-lagged boundary conditions an unfair advantage. Some flow features whose frequencies are not related to the blade passing frequency, and therefore might cause trouble for the chorochronic periodicity and interface, as it occurred in the DDES simulation, are not accounted for. If the full span was simulated, features like tip clearance effects or end wall effects would appear which might have a frequency not directly related to the one the chorochronic boundary conditions are sampling, hence presenting additional challenges. Therefore, the next step is to perform simulations of the full span where all these effects are accounted for, to be able to asses more thoroughly the validity of the method.

\section{Acknowledgements}

The presented work has been carried out within the frame of the project TurboNoiseBB, which is funded by European Union's Horizon 2020 research and innovation program under grant agreement No. 682690714.

The authors would like to acknowledge the Swedish National Infrastructure for Computing (SNIC) and the Chalmers Centre for Computational Science and Engineering $\left(\mathrm{C}^{3} \mathrm{SE}\right)$ in Sweden, for the computational resources used in order to carry out this project.

\section{References}

[1] Spalart, P. R., "Comments on the Feasibility of LES for Wings, and on Hybrid RANS/LES Approach," Proceedings of First AFOSR International Conference on DNS/LES, 1997, 1997.

[2] Spalart, P. R., Deck, S., Shur, M. L., Squires, K. D., Strelets, M. K., and Travin, A., "A New Version of Detached-Eddy Simulation, Resistant to Ambiguous Grid Densities," Theoretical and computational fluid dynamics, Vol. 20, No. 3, 2006 , p. 181.

[3] Hall, K. C., Thomas, J. P., and Clark, W. S., "Computation of Unsteady Nonlinear Flows in Cascades using a Harmonic Balance Technique,” AIAA journal, Vol. 40, No. 5, 2002, pp. 879-886. 


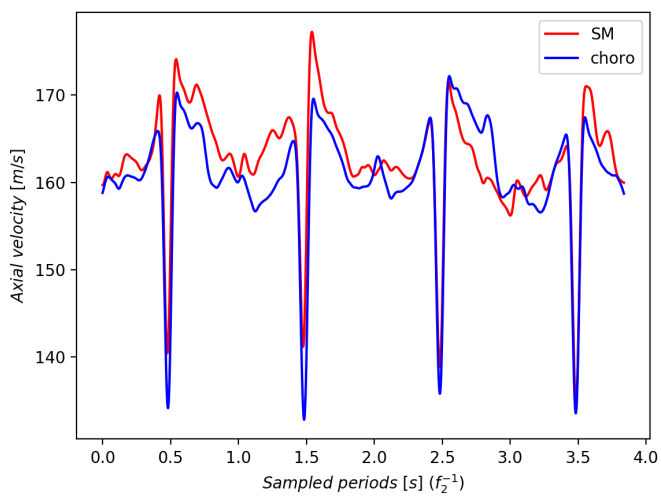

a)

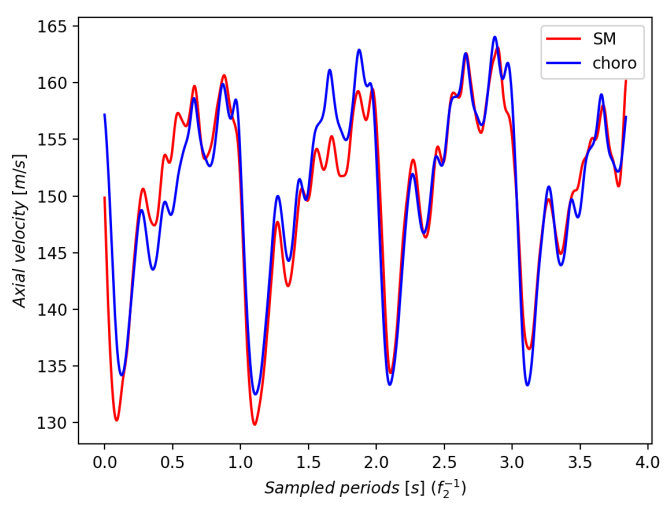

c)

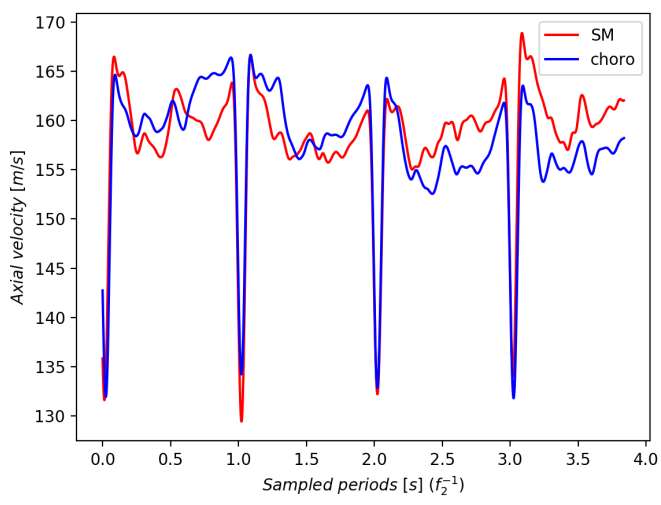

e)

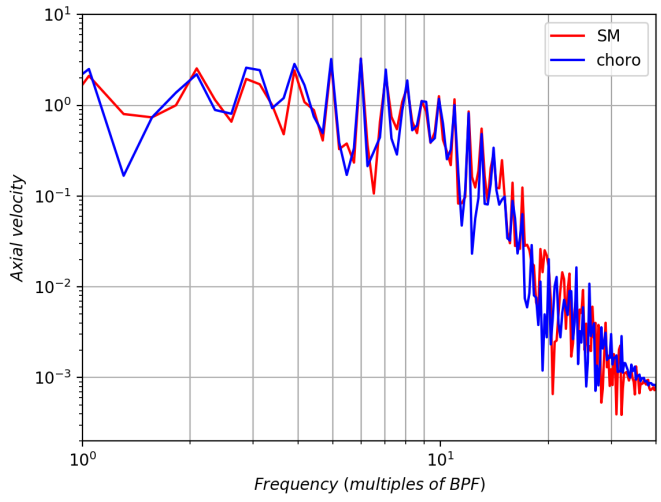

b)

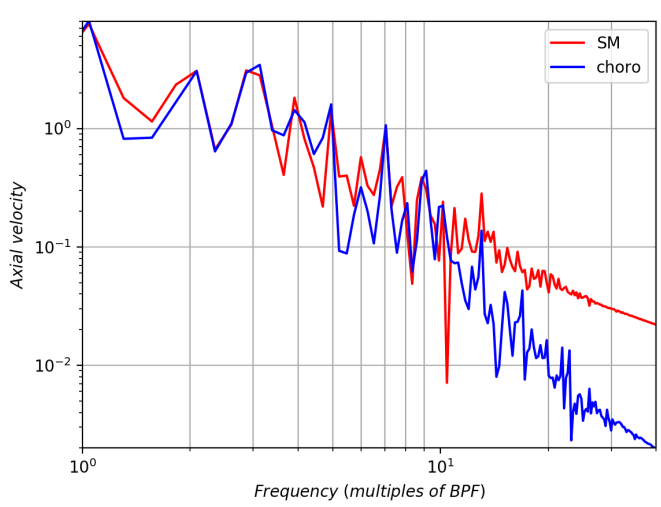

d)

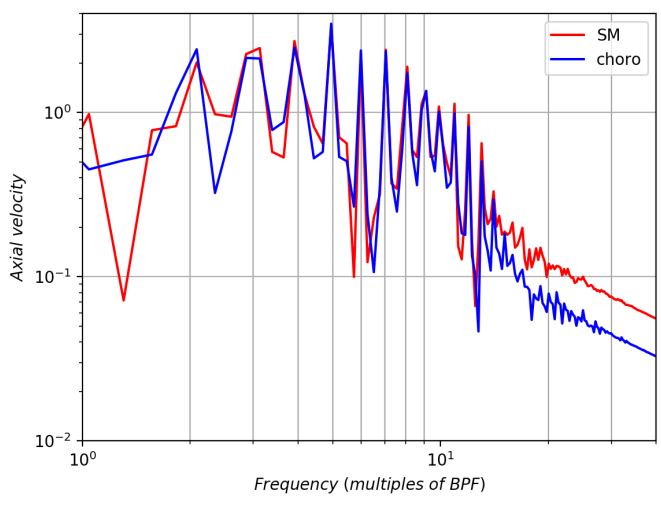

f)

Fig. 11 Comparison of sampled data gathered on points illustrated in Fig. 6 for the DDES simulations. SM and choro refer to the sliding mesh and chorochronic simulation respectively. Axial velocity and its frequency content shown for each point. a) and b) point 1, c) and d) point 2 and e) and f) point 3.

[4] McMullen, M. S., The application of non-linear frequency domain methods to the Euler and Navier-Stokes equations, Citeseer, 2003. 
[5] Ekici, K., and Hall, K. C., "Nonlinear analysis of unsteady flows in multistage turbomachines using harmonic balance," AIAA journal, Vol. 45, No. 5, 2007, pp. 1047-1057.

[6] Weiss, J. M., Subramanian, V., and Hall, K. C., "Simulation of unsteady turbomachinery flows using an implicitly coupled nonlinear harmonic balance method," ASME 2011 Turbo Expo: Turbine Technical Conference and Exposition, American Society of Mechanical Engineers Digital Collection, 2011, pp. 1405-1412.

[7] Sicot, F., Dufour, G., and Gourdain, N., "A time-domain harmonic balance method for rotor/stator interactions," Journal of Turbomachinery, Vol. 134, No. 1, 2012.

[8] Guédeney, T., Gomar, A., Gallard, F., Sicot, F., Dufour, G., and Puigt, G., "Non-uniform time sampling for multiple-frequency harmonic balance computations," Journal of Computational Physics, Vol. 236, 2013, pp. 317-345.

[9] Frey, C., Ashcroft, G., Kersken, H.-P., and Voigt, C., "A harmonic balance technique for multistage turbomachinery applications," ASME Turbo Expo 2014: Turbine Technical Conference and Exposition, American Society of Mechanical Engineers Digital Collection, 2014.

[10] Junge, L., Ashcroft, G., Jeschke, P., and Frey, C., "On the application of frequency-domain methods to multistage turbomachinery," ASME Turbo Expo 2015: Turbine Technical Conference and Exposition, American Society of Mechanical Engineers Digital Collection, 2015.

[11] Crespo, J., Corral, R., and Pueblas, J., "An implicit harmonic balance method in graphics processing units for oscillating blades," Journal of Turbomachinery, Vol. 138, No. 3, 2016.

[12] Campobasso, M. S., and Baba-Ahmadi, M. H., "Analysis of unsteady flows past horizontal axis wind turbine airfoils based on harmonic balance compressible Navier-Stokes equations with low-speed preconditioning," Journal of turbomachinery, Vol. 134, No. 6, 2012.

[13] Erdos, J., Alzner, E., and McNally, W., "Numerical solution of periodic transonic flow through a fan stage," AIAA Journal, Vol. 15, No. 11, 1977, pp. 1559-1568.

[14] He, L., "An Euler Solution for Unsteady Flows Around Oscillating Blades," ASME 1989 International Gas Turbine and Aeroengine Congress and Exposition, American Society of Mechanical Engineers, 1989, pp. V001T01A098-V001T01A098.

[15] Gerolymos, G., Michon, G., and Neubauer, J., "Analysis and Application of Chorochronic Periodicity in Turbomachinery Rotor/Stator Interaction Computations,” Journal of propulsion and power, Vol. 18, No. 6, 2002, pp. 1139-1152.

[16] Gerolymos, G., "Filtered chorochronic interface as a capability for 3-D unsteady throughflow analysis of multistage turbomachinery," International Journal of Computational Fluid Dynamics, Vol. 27, No. 2, 2013, pp. 100-117.

[17] Ben Nasr, N., and Gerolymos, G., "RSM_RANS Computation of Aircraft Engine Fan/OGV Unsteady Aerodynamics for Noise-Prediction Input," 15th AIAA/CEAS Aeroacoustics Conference (30th AIAA Aeroacoustics Conference), 2009 , p. 3151.

[18] Connell, S., Braaten, M., Zori, L., Steed, R., Hutchinson, B., and Cox, G., "A Comparison of Advanced Numerical Techniques to Model Transient Flow in Turbomachinery Blade Rows," ASME 2011 Turbo Expo: Turbine Technical Conference and Exposition, American Society of Mechanical Engineers, 2011, pp. 1241-1250.

[19] Schnell, R., "Investigation of the tonal acoustic field of a transonic fanstage by time-domain CFD-calculations with arbitrary blade counts," ASME Turbo Expo 2004: Power for Land, Sea, and Air, American Society of Mechanical Engineers Digital Collection, 2004, pp. 1763-1773.

[20] He, L., "Fourier methods for turbomachinery applications," Progress in Aerospace Sciences, Vol. 46, No. 8, 2010 , pp. $329-341$.

[21] Mouret, G., Gourdain, N., and Castillon, L., "Adaptation of Phase-Lagged Boundary Conditions to Large Eddy Simulation in Turbomachinery Configurations," Journal of Turbomachinery, Vol. 138, No. 4, 2016, p. 041003.

[22] Burak, M., Large Eddy Simulation for the Analysis of Supersonic Jet Noise Suppression Devices, Chalmers University of Technology, 2010.

[23] Tyler, J. M., and Sofrin, T. G., "Axial flow compressor noise studies,” Tech. rep., SAE Technical Paper, 1962.

[24] Olausson, M., Eriksson, L.-E., and Baralon, S., "Evaluation of nonlinear rotor wake/stator interaction by using time domain chorochronic solver," Proceedings of the 8th International Symposium on Experimental and Computational Aerothermodynamics of Internal Flows, Lyon, 2007. 
[25] Biesinger, T., Cornelius, C., Rube, C., Braune, A., Campregher, R., Godin, P. G., Schmid, G., and Zori, L., "Unsteady CFD methods in a commercial solver for turbomachinery applications," ASME Turbo Expo 2010: Power for Land, Sea, and Air, American Society of Mechanical Engineers Digital Collection, 2010, pp. 2441-2452.

[26] Eriksson, L., "Development and validation of highly modular flow solver versions in g2dflow and g3dflow," Volvo Aero Corporation, Trollhättan, Sweden, Technical Report, , No. 9970-1162, 1995.

[27] Spalart, P. R., and Allmaras, S. R., "A One Equation Turbulence Model for Aerodynamic Flows," 30th AIAA Aerospace Sciences Meeting and Exhibit, AIAA Paper 92-0439, 1992.

[28] Shur, M. L., Spalart, P. R., Strelets, M. K., and Travin, A. K., "An enhanced version of DES with rapid transition from RANS to LES in separated flows," Flow, turbulence and combustion, Vol. 95, No. 4, 2015, pp. 709-737.

[29] Siggeirsson, E. M., and Andersson, N., "The NASA 2D wall-mounted hump simulated using DDES-SA with the G3D:: Flow solver," AIAA Scitech 2019 Forum, 2019, p. 0083.

[30] Ashton, N., West, A., and Mendonça, F., "Flow dynamics past a 30P30N three-element airfoil using improved delayed detached-eddy simulation," AIAA Journal, 2016, pp. 3657-3667.

[31] Siggeirsson, E. M., Andersson, N., and Wallin, F., "Sensitivity study of the SA-DDES shielding function," 2018 AIAA Aerospace Sciences Meeting, 2018, p. 1355.

[32] Probst, A., Wolf, C., Radespiel, R., Knopp, T., Schwamborn, D., and Radespiel, R., "A Comparison of Detached-Eddy Simulation and Reynolds-Stress Modeling Applied to the Flow over a Backward-Facing Step and an Airfoil at Stall," 48th AIAA Aerospace Sciences Meeting Including the New Horizons Forum and Aerospace Exposition, 2010, p. 920.

[33] Lejon, M., Grönstedt, T., Glodic, N., Petrie-Repar, P., Genrup, M., and Mann, A., "Multidisciplinary design of a three stage high speed booster," ASME Turbo Expo 2017: Turbomachinery Technical Conference and Exposition, American Society of Mechanical Engineers Digital Collection, 2017.

[34] “GitHub repository for VINK," 2017. https://github.com/nikander/VINK 